\title{
Envelhecimento ativo, qualidade de vida e cognição de idosos: um estudo transversal em uma cidade de Minas Gerais
}

\author{
Active aging, quality of life and cognition of elderly: a cross-sectional study in a city of \\ Minas Gerais
}

Envejecimiento activo, calidad de vida y cognición de las personas mayores: un estudio transversal en una ciudad de Minas Gerais

Jaqueline Gabriele Silva¹, Caio Godinho Caldeira', Gylce Eloísa Cabreira Panitz Cruz¹, Luciana Estefani Drumond de Carvalho ${ }^{1 *}$.

\section{RESUMO}

Objetivos: Avaliar a associação entre a cognição, a prática de atividade física, a qualidade de vida e investigar a influência dos fatores sociodemográficos em idosos em uma cidade de médio porte no estado de Minas Gerais. Métodos: $O$ estudo foi observacional, transversal e quantitativo. Foram entrevistados 303 idosos. Utilizaram-se uma ficha de identificação, o teste de qualidade de vida WHOQOL-OLD, o Questionário Internacional de Atividade Física (IPAQ) e o Mini-exame do Estado Mental (MEEM). Para a análise, foram aplicados os testes de Kruskal-Wallis e de Mann-Whitney, com significância de $p<0,05$. Resultados: Ressalta-se a predominância do sexo feminino, na faixa etária de 66 a 70 anos, com escolaridade entre 4 e 8 anos e renda de até dois salários mínimos. A amostra apresentou cognição majoritariamente preservada ( $n=186 ; 61 \%)$, qualidade de vida regular $(n=163 ; 54 \%)$ e se mostrou ativa quanto ao nível de atividade física $(n=251 ; 83 \%)$. Idosos ativos apresentaram melhor qualidade de vida, entretanto não houve associação com melhora da cognição. Observou-se que maior idade, menor renda e menor escolaridade se correlacionam com menor nível cognitivo. Conclusão: Notou-se relação positiva entre a qualidade de vida dos idosos e a prática de atividade física.

Palavras-chave: Cognição, Atividade física, Qualidade de vida, Envelhecimento.

\begin{abstract}
Objectives: To evaluate the association between cognition, physical activity, and quality of life and to investigate the influence of sociodemographic factors in the elderly in a medium-sized city in the state of Minas Gerais. Methods: The study was observational, transversal and quantitative. A total of 303 elderly people were interviewed. We used an identification form, the WHOQOL-OLD quality of life test, the International Physical Activity Questionnaire (IPAQ) and the Mini-Mental State Examination (MMSE). For the analysis, the Kruskal-Wallis and Mann-Whitney tests were applied, with significance of $p<0.05$. Results: The predominance of females in the age group of 66 to 70 years, with education between 4 and 8 years and income of up to two minimum wages is noteworthy. The sample presented mostly preserved cognition $(n=186 ; 61 \%)$, regular quality of life $(n=163 ; 54 \%)$ and was active in terms of level of physical activity ( $n=251 ; 83 \%)$. Active elderly individuals presented better quality of life, however, there was no association with improvement in cognition. It was observed that higher age, lower income and lower education correlate with lower cognitive level. Conclusion: There was a positive relationship between the quality of life of the elderly and the practice of physical activity.
\end{abstract}

Keywords: Cognition, Physical activity, Quality of life, Aging.

${ }^{1}$ Universidade Federal de São João Del-Rei, Campus Centro-Oeste, Divinópolis-MG.

*E-mail: lucianadrumond@ufsj.edu.br

SUBMETIDO EM: 9/2019

ACEITO EM: 10/2019

PUBLICADO EM: 1/2020

REAS/EJCH | Vol.12(1) | e1796 | DOI: https://doi.org/10.25248/reas.e1796.2020 Página 1 de 10 


\section{RESUMEN}

Objetivos: Evaluar la asociación entre cognición, actividad física y calidad de vida e investigar la influencia de los factores sociodemográficos en los ancianos de una ciudad mediana del estado de Minas Gerais. Métodos: El estudio fue observacional, transversal y cuantitativo. Se entrevistó a un total de 303 personas mayores. Utilizamos un formulario de identificación, la prueba de calidad de vida WHOQOL-OLD, el Cuestionario Internacional de Actividad Física (IPAQ) y el Mini-Mental State Examination (MMSE). Para el análisis se aplicaron las pruebas de Kruskal-Wallis y Mann-Whitney, con una significación de p<0,05. Resultados: Cabe destacar el predominio de las mujeres en el grupo de edad de 66 a 70 años, con educación entre 4 y 8 años e ingresos de hasta dos salarios mínimos. La muestra presentaba principalmente cognición preservada $(n=186 ; 61 \%)$, calidad de vida regular $(n=163 ; 54 \%)$ y era activa en términos de nivel de actividad física $(n=251 ; 83 \%)$. Los ancianos activos presentaron una mejor calidad de vida, sin embargo, no hubo asociación con mejoría en la cognición. Se observó que la mayor edad, los menores ingresos y la menor educación se correlacionan con un nivel cognitivo más bajo. Conclusión: Había una relación positiva entre la calidad de vida de los ancianos y la práctica de la actividad física.

Palabras-clave: Cognición, Actividad física, Calidad de vida, Envejecimiento.

\section{INTRODUÇÃO}

A definição de idoso engloba os indivíduos a partir de 60 anos, para os países subdesenvolvidos e 65 anos, para os países desenvolvidos. No Brasil, a Política Nacional do Idoso estabelece como idoso o indivíduo com 60 anos ou mais (BRASIL, 1994).

Um crescimento da população idosa está se estabelecendo, tanto em âmbito nacional quanto em mundial (CARVALHO A, et al., 2014). A evolução da composição populacional indica uma tendência de envelhecimento demográfico, que equivale ao aumento da participação percentual dos idosos na população e a paralela redução dos outros grupos etários. Analisando-se projeções populacionais, identifica-se um crescimento exorbitante quanto à participação dos idosos de 60 anos de idade ou mais na população brasileira, que representavam 11,7\% do total em 2015 e equivalerão a mais de 35\%, em 2070 (IBGE, 2016). Essa expressiva progressão numérica decorre em consequência de alguns fatores, destacando-se a diminuição da natalidade e o aumento da expectativa média de vida (DANIEL F, et al., 2018).

O envelhecimento deve ser entendido como um processo multidimensional e multideterminado, de caráter progressivo e gradual, acompanhado por inúmeras mudanças de cunho biológico, psicológico e social. Está atrelado ao declínio natural de funções fisiológicas que influenciam o indivíduo, sendo marcante uma acentuação exponencial da fragilidade e de várias doenças físicas. Além disso, evidencia-se a necessidade de lidar com recorrentes perdas, a redução da plasticidade comportamental e das interações sociais, o aumento da seletividade dos relacionamentos sociais e as modificações emocionais (DANIEL F, et al., 2018).

Dentre as diversas ocorrências características do avanço da idade, uma das mais importantes é o declínio no desempenho cognitivo (SOARES RM, et al., 2013). A cognição é fruto do processamento coordenado entre áreas cerebrais distribuídas, sendo que interrupções na comunicação dessas redes neurais podem resultar em disfunção cognitiva. Dessa forma, a desconexão cortical contribui para o declínio em alguns aspectos do funcionamento cognitivo identificado no envelhecimento saudável (BENNETT IJ, et al., 2009), resultando em alterações entre os principais indicadores de desempenho cognitivo, sendo eles: a atenção, a memória episódica, a memória de trabalho, a memória semântica e função executiva (SOARES RM, et al., 2013).

É de suma importância compreender as mudanças cognitivas associadas ao envelhecimento, tanto normal quanto patológico. Por mais que a demência e o comprometimento cognitivo leve sejam comuns, há também alterações cognitivas sutis associadas ao envelhecimento, que podem ser experimentadas por qualquer indivíduo. Elas devem ser entendidas, pois podem afetar o cotidiano de um idoso e, ademais, nos ajudar a distinguir estados normais de patologias (HARADA CN, et al., 2013). 
Dada a expansão da população idosa, a prevenção do declínio cognitivo em idosos saudáveis requer destaque (NAQVI R, et al. 2013). Nesse sentido, a análise da literatura existente demonstra que idosos que mantêm um desempenho cognitivo normal, em geral, são idosos mais jovens, possuem níveis mais elevados de escolaridade e renda, apresentam maiores níveis de participação em comunidade e realizam atividades que envolvem estimulação física, mental e social (DIAS EC, et al., 2015). Muitos estudos defendem que a prática de atividade física moderada ou intensa no envelhecimento pode ser um tratamento não farmacológico para melhora da função cognitiva dos idosos (OLIVEIRA D, et al., 2018). Demonstra-se que ela pode ter efeitos protetores e regenerativos contra o declínio cognitivo, estando, também, associada a benefícios na atenção, na função executiva (SATOH M, et al., 2014) e na memória de curto prazo (OLIVEIRA D, et al., 2018).

A prática de exercício físico, quando regular, desenvolve alterações positivas que desencadeiam a angiogênese e o aumento do fluxo sanguíneo cerebral, a sinaptogênese e a neurogênese, o que é extremamente favorável para o indivíduo (OLIVEIRA D, et al., 2018). Embora várias pesquisas indiquem essa relação de influência entre atividade física e desempenho cognitivo de idosos, esses resultados não são consensuais (SOARES RM, et al., 2013). Alguns autores sugerem que a atividade física pode contribuir, também, para uma melhor qualidade de vida (BLESSMANN EJ, et al., 2005), simultâneo a melhoria de desempenho cognitivo já mencionado (LANGOIS F e VU T 2013).

Estudos recentes sugerem que as intervenções de treinamento físico também podem levar a uma evolução significativa no rendimento cognitivo e promover ganho de qualidade de vida (LANGOIS F e VU T, 2013). Sabe-se que essas esferas estão relacionadas, tendo em vista que o déficit cognitivo perturba a qualidade de vida do idoso por conduzir à perda de sua autonomia e independência (SILVEIRA MM, 2015). Ao se considerar a possibilidade de redução do declínio cognitivo em idosos, faz-se necessário conhecer os fatores que estão associados à ocorrência desse processo, influenciando-o em diferentes escalas. Por meio desse conhecimento, é possível estabelecer-se estratégias e ações que favoreçam o bem-estar desse vulnerável grupo da sociedade. Diante do exposto, esse estudo visou avaliar a associação entre o desempenho cognitivo e a qualidade de vida, a prática de atividades físicas e fatores sociodemográficos de idosos residentes em uma cidade de médio porte do estado de Minas Gerais.

\section{MÉTODOS}

Trata-se de um estudo observacional, analítico, do tipo transversal e quantitativo. A população amostral abrangeu os idosos de uma cidade do interior do estado de Minas Gerais. De acordo com o censo do Instituto Brasileiro de Geografia e estatística - IBGE (2010), a população total do município era de 213.000 habitantes, deste total $7,6 \%$ são considerados idosos, ou seja, 16.189 pessoas. O número de participantes da pesquisa foi determinado, mediante cálculo, utilizando a estimativa de erro amostral para tal população de idosos, resultando num total mínimo de 303. O tipo de amostragem utilizado foi aleatório simples.

A coleta de dados foi realizada em quatro locais, no período de outubro de 2016 a novembro de 2017 , abrangendo o total de 303 idosos: duas instituições religiosas da cidade onde foi desenvolvido o estudo, um centro de convivência de idosos da mesma cidade e com os idosos de um programa voltado a essa população no campus de uma universidade federal localizado no município. Foram realizadas reuniões com os responsáveis pelos pontos de coleta, em que se detalharam todas as etapas e objetivos do projeto. Após a anuência destes, a equipe de pesquisadores fez um convite aos idosos presentes nos locais de coleta, a fim de que estes participassem como voluntários.

O critério de inclusão foi idade igual ou superior a 60 anos - caracterizando o sujeito como idoso (BRASIL, 1994; BRASIL, 2016). Como critérios de exclusão, determinou-se: limitações, como afasia e ventilação mecânica, que impossibilitassem as respostas às questões propostas nos instrumentos utilizados. Os participantes preenchiam uma ficha com dados sociodemográficos e três instrumentos eram, então, aplicados por acadêmicos voluntários previamente treinados pelos pesquisadores: o Questionário Internacional de Atividade Física (IPAQ), o questionário de qualidade de vida da Organização Mundial da Saúde WHOQOLOLD e o Mini-exame do Estado Mental (MEEM). 
O Mini-exame do Estado Mental (MEEM) é um instrumento utilizado para uma avaliação cognitiva geral. O teste pode ser avaliado de 0 a 30 pontos, sendo que os critérios utilizados para definir uma possível limitação cognitiva são: analfabetos $\leq 17$ pontos; alguma escolaridade $\leq 24$ pontos. Apresenta 20 questões, cada qual com a sua respectiva pontuação, que avaliam os seguintes aspectos: memória recente, memória imediata, orientação espacial e temporal, atenção, linguagem, cálculo e habilidade construcional (LOURENÇO RA, et al., 2006).

O Questionário Internacional de Atividade Física (IPAQ) é um instrumento que permite estimar o tempo semanal gasto na realização de atividades físicas de intensidade moderada a vigorosa e em diferentes contextos da vida (trabalho, tarefas domésticas, transporte e lazer). O teste é dividido em quatro domínios: atividades físicas como trabalho, como transporte, domésticas e lazer/exercícios físicos. São investigados, então, o tempo diário e a frequência semanal de cada atividade e, de acordo com uma tabela padronizada para a interpretação de tais valores, os idosos são classificados em sedentários, irregularmente ativos, ativos ou muito ativos (BENEDETTI TRB, et al., 2007).

Para a avaliação da qualidade de vida dos sujeitos da pesquisa, o instrumento selecionado foi o questionário de qualidade de vida desenvolvido pelo World Health Organization Quality of Life Group (WHOQOL-OLD), que é constituído de 24 questões correspondentes a seis domínios: funcionamento do sensório; autonomia; atividades passadas, presentes e futuras; participação social; morte e morrer; intimidade. Cada pergunta apresenta um score de 1 a 5 pontos, o qual é somado posteriormente. Faz-se então a média desses valores e o indivíduo é classificado com qualidade de vida ruim de 0 - 2,99 pontos, regular de 3 a 3,99, boa qualidade de vida entre 4 a 4,99 e muito boa com 5 pontos. (ORGANIZAÇÃO MUNDIAL DE SAÚDE, 2005).

Quanto à análise, os dados coletados foram armazenados e convertidos em planilhas no programa Microsoft Excel 2010, permitindo uma análise descritiva. A análise estatística foi realizada com o auxílio do software GhaphPad Prism, onde foram confeccionados gráficos e tabelas. Para verificar a distribuição da amostra, foi utilizado o teste de Kolmogorov-Smirnov. Como as variáveis se mostraram não paramétricas, os dados foram descritos como mediana e os testes estatísticos utilizados foram: Kruskall-Wallis ou MannWhitney. Os valores foram considerados estatisticamente significativos quando $p<0,05 /$ intervalo de confiança de $95 \%$.

O estudo foi submetido à análise do Comitê de Ética em Pesquisa da Universidade Federal de São João del Rei - Campus Centro-Oeste e aprovado com o parecer n¹.686.005 e CAAE 57380816.0.0000.5545. Aos participantes foi apresentado o Termo de Consentimento Livre e Esclarecido (TCLE), garantindo a confidencialidade e privacidade, conforme os princípios dispostos na Resolução no 466/2012 do Conselho Nacional de Saúde.

\section{RESULTADOS}

Foram entrevistados 303 idosos em diversos pontos de coleta do município avaliado. A amostra foi constituída de $(n=236 ; 78 \%)$ mulheres e $(n=67 ; 22 \%)$ homens. Quanto à faixa etária, $(n=61 ; 20 \%)$ se encontravam na faixa de 60-65 anos; $(n=95 ; 31 \%)$, na de 66-70 anos; $(n=72 ; 24 \%)$ na de $71-75$ anos; $(n=41$; $14 \%)$ na de $76-80$ anos e $(n=34 ; 11 \%)$ com mais de 80 anos. Quanto à escolaridade, observou-se: $(n=9 ; 3 \%)$ indivíduos analfabetos; $(n=93 ; 31 \%)$ com $0-3$ anos de estudo; $(n=134 ; 44 \%)$ com $4-8$ anos de estudo e ( $n=66$; $22 \%)$ com mais de 8 anos de estudo. Quanto à renda, $(n=172 ; 57 \%)$ relataram possuir renda de até 2 salários mínimos; ( $n=89 ; 29 \%)$, mais que 2 até 4 salários; $(n=29 ; 10 \%)$, mais que 4 até 10 salários; $(n=9 ; 3 \%)$, mais que 10 até 20 salários e $(n=3 ; 1 \%)$, mais que 20 salários. Ressalta-se a predominância do sexo feminino, na faixa etária de 66 a 70 anos, com escolaridade entre 4 e 8 anos de estudo e renda de até dois salários mínimos.

Quanto aos parâmetros cognitivos, a amostra foi majoritariamente classificada como normal ( $n=186 ; 61 \%)$. Já, quanto aos escores de qualidade de vida, a maioria obteve classificação regular $(n=163 ; 54 \%)$. E quanto aos níveis de atividade física, a maior parte dos entrevistados foi considerada ativa $(n=251 ; 83 \%)$, conforme Tabela 1. 
Tabela 1 - Perfil cognitivo, de atividade física e qualidade de vida.

\begin{tabular}{cccc}
\hline Parâmetro avaliado & Características & No & $\%$ \\
\hline \multirow{2}{*}{ Avaliação cognitiva geral (meem) } & Normal & 186 & $61 \%$ \\
& Alterado & 117 & $39 \%$ \\
& Sedentário & 4 & $1 \%$ \\
\hline \multirow{2}{*}{ Nível de atividade física (ipaq) } & Irregularmente ativo & 27 & $9 \%$ \\
& Ativo & 251 & $83 \%$ \\
& muito ativo & 21 & $7 \%$ \\
\hline \multirow{2}{*}{ Qualidade de vida (whoqol-old) } & Ruim & 9 & $3 \%$ \\
& Regular & 163 & $54 \%$ \\
& Boa & 131 & $43 \%$ \\
\hline
\end{tabular}

Fonte: Silva JG, et al., 2019.

Relacionando-se esses parâmetros, obteve-se que indivíduos com idade mais avançada (mais de 80 anos) apresentaram nível cognitivo menor $(22,7 ; n=34)$ quando comparados àqueles mais jovens de 60 a 65 anos (25; $n=61)$, como demonstrado no Gráfico 1-A. Percebe-se, também, que indivíduos com renda de até dois salários mínimos apresentam nível cognitivo mais baixo $(23,1 ; n=172)$ em comparação com aqueles que apresentam renda de 2 a 4 salários mínimos $(25,1 ; n=89)$ ou de 4 a $10(26,5 ; n=29)$, conforme evidenciado no Gráfico 1-B. Já, considerando a escolaridade, observa-se que, quanto maior a escolaridade, maior o nível cognitivo da amostra avaliada (Gráfico 1-C).

Gráfico 1 - Cognição e fatores sociodemográficos. Os dados estão representados como a mediana do índice obtido no MEEM.

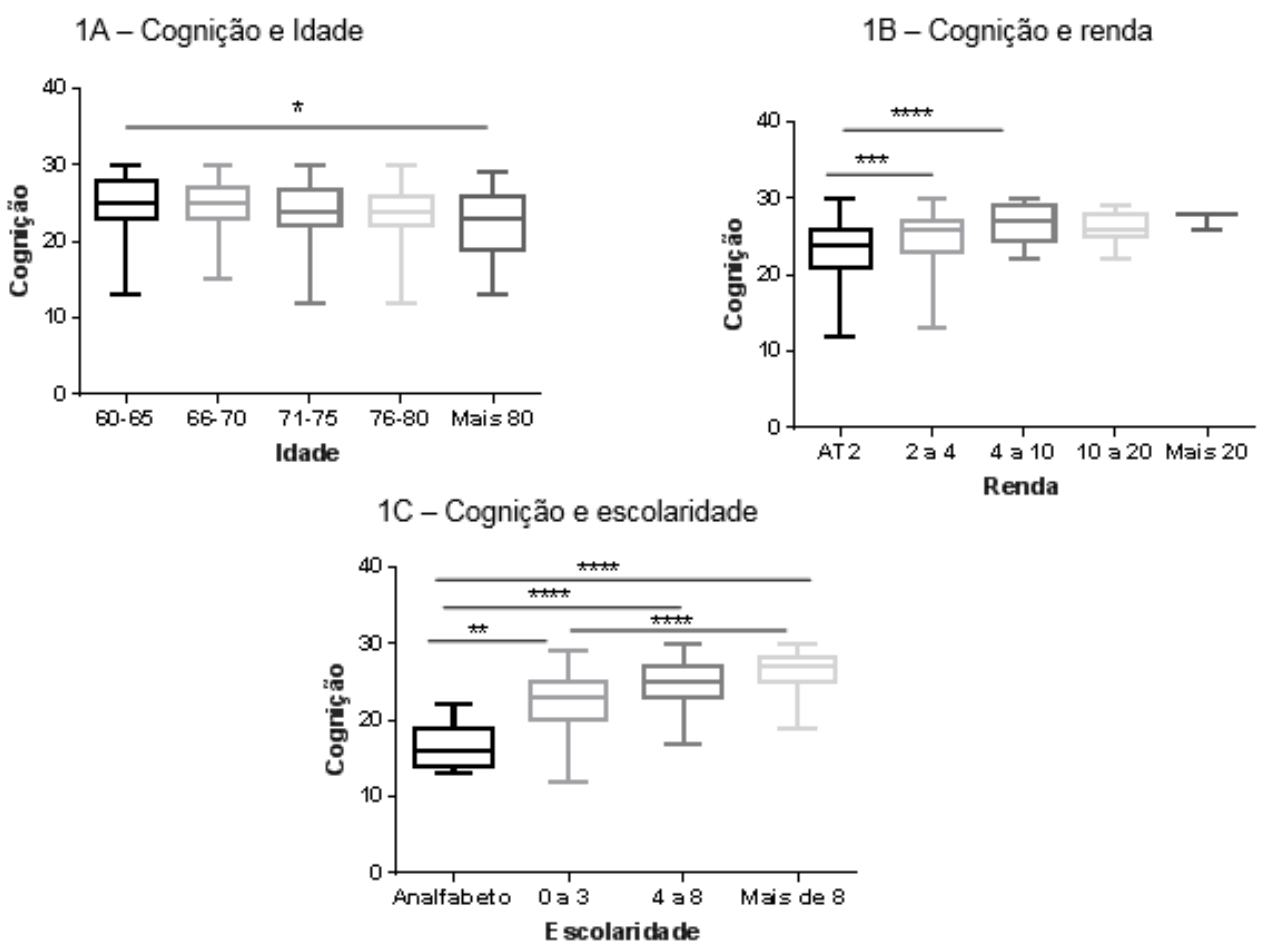

Legenda: (1A) Cognição e idade. Os dados estão representados como mediana. 60-65= 25 ( $n=61$ ), 66-70= 24,5 $(n=95), 71-75=23,9(n=72), 76-80=23,4(n=41)$ e mais $80=22,7(n=34)$. (1B) Cognição e renda. Os dados estão representados como mediana. AT2=23,1 ( $n=172), 2$ a $4=25,1(n=89), 4$ a $10=26,5(n=29), 10$ a $20=26,3(n=9)$ e mais $20=27,3(n=3)$. (1C) Cognição e escolaridade. Os dados estão representados como mediana. Analfabeto= $16,4(n=9), 0$ a $3=22,3(n=93), 4$ a $8=24,8(n=134)$ e mais de $8=26,3(n=66)$. KRUSKAL-WALLIS ${ }^{*} p<0,05$.

Fonte: Silva JG, et al., 2019. 
Não se encontrou relação entre os níveis cognitivos, os parâmetros de qualidade de vida e os níveis de atividade física (Gráficos 2 e 3).

Gráfico 2 - Qualidade de vida e atividade física.

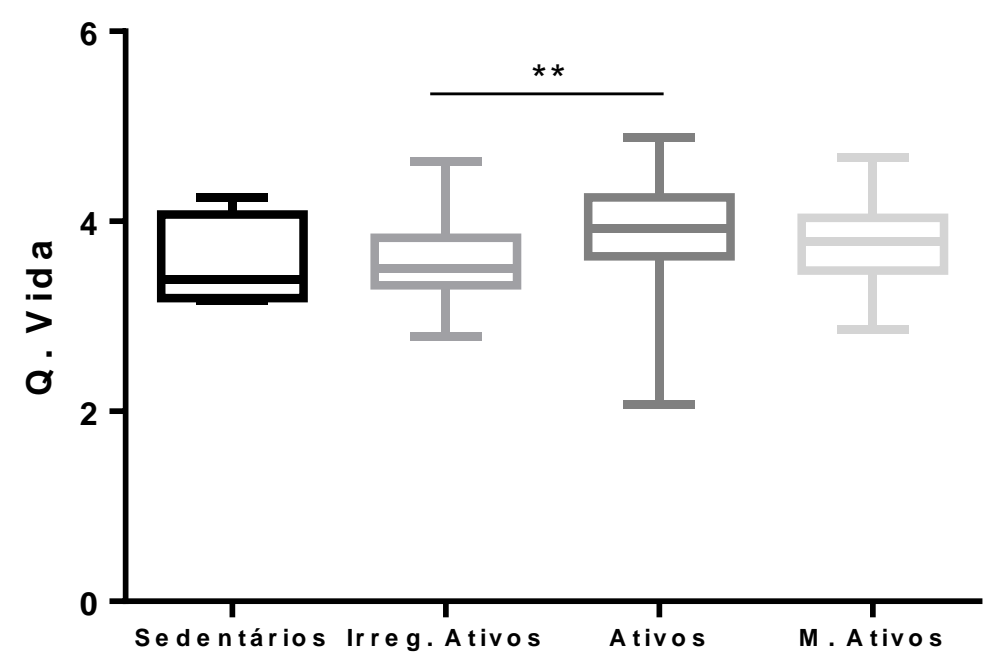

Atividade Física

Legenda: Qualidade de vida e atividade física. Os dados estão representados como mediana. Sedentários $=3,6(n=4)$, irregularmente ativos $=3,6(n=27)$, Ativos $=3,9(n=251)$ e M. Ativos $=3,8(n=21)$. KRUSKAL-WALLIS ${ }^{*} \mathrm{P}<0,05$.

Fonte: Silva JG, et al., 2019.

Gráfico 3 - Cognição e atividade física.

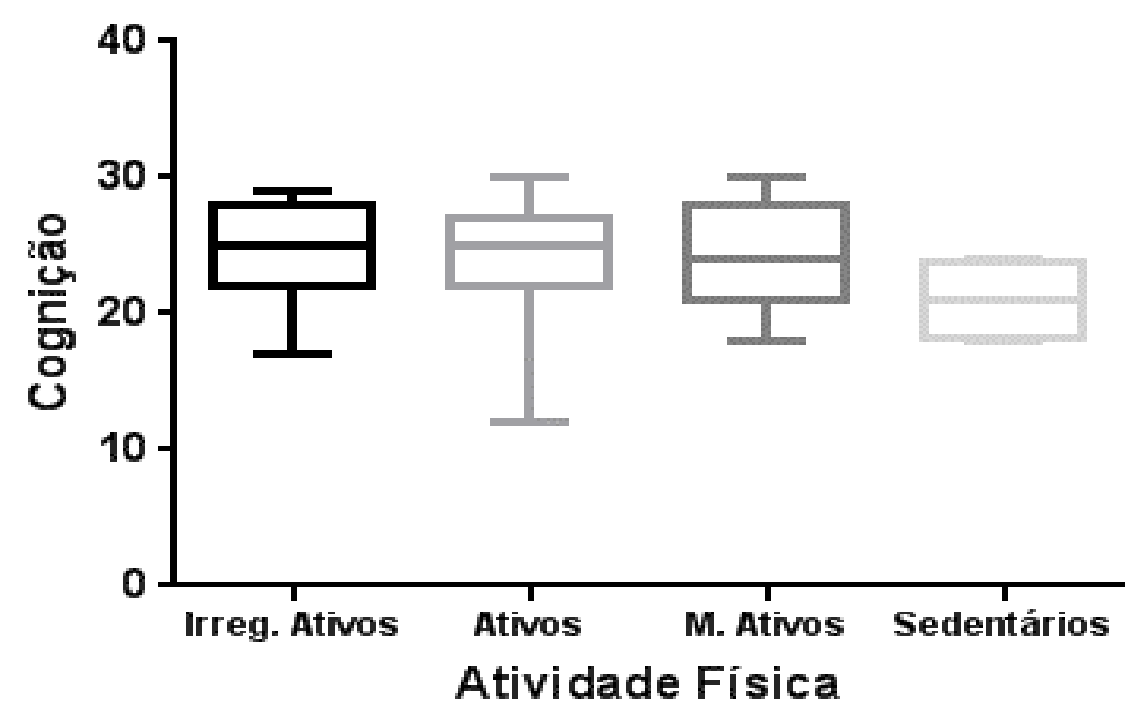

Legenda: Cognição e atividade física. Os dados estão representados como mediana. Irreg. Ativos = 24,3 $(n=27)$, Ativos $=24,1 \pm 0,2 \quad(n=251)$, M. Ativos $=24,6(n=21)$ e Sedentários $=21 \quad(n=4)$. KRUSKAL-WALLIS ${ }^{*} p>0,05$.

Fonte: Silva JG, et al., 2019. 
Quanto aos níveis de atividade física, percebe-se que indivíduos classificados como fisicamente ativos apresentam qualidade de vida melhor que aqueles classificados como sedentários ou irregularmente ativos, conforme Gráfico 4.

Gráfico 4 - Cognição e Qualidade de Vida.

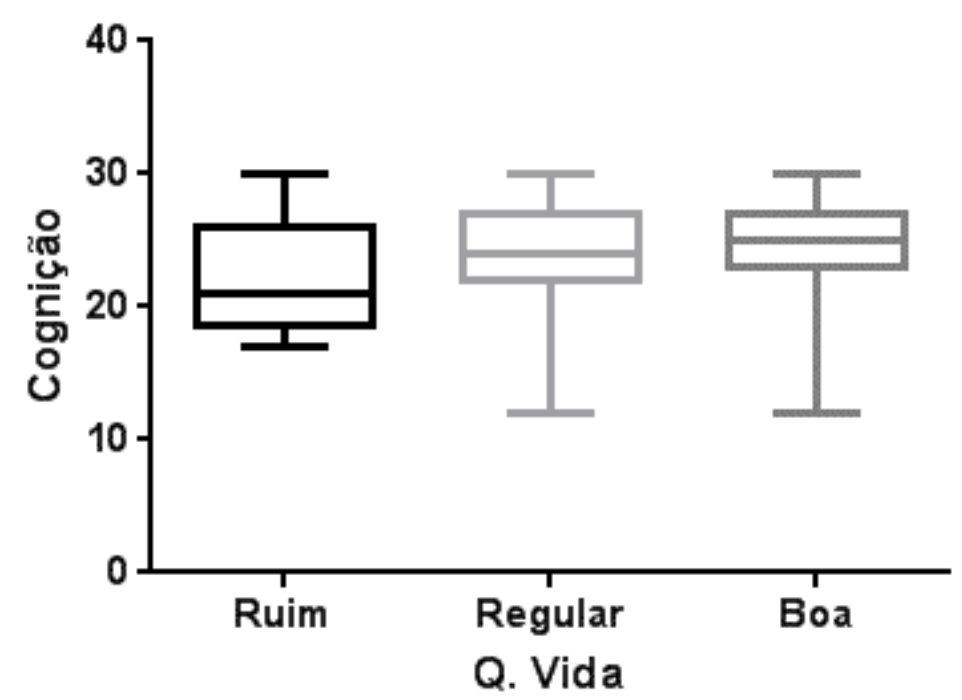

Legenda: Os dados estão representados como mediana. Ruim=22,1 $(n=9)$, Regular=23,9 ( $n=163)$, Boa = 24,6 ( $n=131)$.

Fonte: Silva JG, et al., 2019.

\section{DISCUSSÃo}

O presente estudo identificou um perfil sociodemográfico predominante entre os idosos condizente com a literatura científica prevalente. A superioridade numérica de indivíduos do sexo feminino observada vai ao encontro do processo da feminização da velhice, concordando com o dado de que as mulheres constituem a maioria da população idosa em todas as regiões do mundo, vivendo, em média, de cinco a sete anos a mais que os homens (ALMEIDA AV, et al., 2015).

A discreta maioria de idosos até 70 anos observada, correspondendo a $51 \%$ da amostra total, é próxima ao encontrado por Luz EP, et al (2014), de 50,2\%, e indica o importante crescimento da população idosa de 70 anos de idade ou mais. Enfatiza-se que, o considerável aumento de idosos mais velhos exige uma reorganização das políticas públicas, estabelecendo-se estratégias direcionadas às necessidades da população. Ainda sobre o perfil sociodemográfico, constatou-se, majoritariamente, rendas de até 2 salários mínimos entre os idosos, assim como notado no citado estudo de Luz EP, et al (2014), que justificou a renda mensal relativamente baixa por suas fontes predominantes, sendo destaques aposentadorias e pensões. Quanto à escolaridade, encontrou-se $78 \%$ dos indivíduos com menos de 8 anos de estudo, compatível com o trabalho de Castro C, et al (2018), que com uma amostra de 11.177 idosos identificou esse mesmo grau de escolaridade em $77,7 \%$ dos participantes.

Os achados, a partir da análise dos fatores sociodemográficos relacionados ao desempenho cognitivo, corroboram com diversas pesquisas anteriores. A influência do nível de escolaridade e da idade no declínio cognitivo em idosos tem sido uma associação encontrada em diversos estudos populacionais, no Brasil e em outros países em desenvolvimento. Em estudo realizado em Santo Antônio de Pádua, com 341 idosos, constatou-se que a escolaridade e a idade exercem influência expressiva nas pontuações do MEEM. Da mesma forma, um estudo desenvolvido na Santa Casa de São Paulo, com 211 idosos, confirmou que a idade e a escolaridade se associam de forma significativa com o rendimento dos idosos no MEEM (MACHADO JC, et al., 2018). 
Ambos corroboram com os resultados encontrados, reforçando que os níveis cognitivos mais elevados são identificados nos idosos mais jovens e com mais anos de estudo. Ademais, a constatação de que uma renda mais baixa associa-se ao menor desempenho cognitivo, observada no presente estudo, também foi verificada em pesquisa desenvolvida com 180 alunos da Universidade Aberta da Terceira Idade da Universidade de São Paulo, na qual percebeu-se associação positiva significativa entre o fator renda e a performance no exame de cognição (KISSAKI PT, et al., 2010).

Ainda que muitos estudos estabeleçam a prática de atividade física moderada ou intensa no envelhecimento como um possível recurso para melhoria da função cognitiva dos idosos (OLIVEIRA D, et al., 2018), não se encontrou relação entre os níveis cognitivos e os níveis de atividade física. Da mesma forma, não houve relação entre os níveis cognitivos e os parâmetros de qualidade de vida. Esses achados conflituosos podem ser justificados pela utilização do instrumento MEEM. Sabe-se que os estudos com seu emprego têm sido realizados, preponderantemente, com amostras advindas de grandes cidades do Sudeste, o que difere da amostra utilizada nesse estudo, recrutada em uma cidade de médio porte (MELO DM e BARBOSA AJG, 2015). Apesar de ser validado para utilização no Brasil, as características psicométricas do MEEM devem ser investigadas nas diversas regiões, tendo em vista as pluralidades social, cultural e econômica, características evidentes do país. Portanto, é importante analisar a confiabilidade dessa ferramenta considerando-se, especificamente, o perfil da população a ser investigada, visando-se estimar a estabilidade do instrumento. Por meio da análise da confiabilidade é possível identificar até que ponto os resultados poderão ser, de fato, considerados seguros e poderão ser generalizados a todos os casos a que forem aplicados (SANTOS S, et al., 2010). Uma das limitações do presente estudo foi a não realização da análise de confiabilidade para população de idosos da cidade de Divinópolis.

Ressalta-se que o uso do MEEM no Brasil enfrenta inúmeras dificuldades além da heterogeneidade evidente na população. Há, ainda, escassez de evidências de validade do instrumento nesse contexto, além de críticas quanto a não existência de uniformidade no processo de aplicação e avaliação do teste, consequência da variedade de versões, adaptações e pontos de corte existentes. Ademais, a presença de itens que demandam educação formal torna ainda mais desafiador a detecção de disfunções cognitivas, tendo em vista que na realidade do país há expressiva parcela da população com pouca escolarização. Por fim, questionamentos sobre a maneira como o MEEM avalia determinadas funções, como a memória de trabalho, feita com base em somente uma questão, corroboram com a possibilidade de resultados duvidosos para declínio cognitivo a partir desse instrumento. A despeito das dificuldades enfrentadas para uso do MEEM no Brasil, sua utilização tem sido significativa tanto na clínica quanto na pesquisa (MELO DM e BARBOSA AJG, 2015).

Além disso, a literatura aponta que idosos utilizadores das tecnologias de informação podem apresentar benefícios cognitivos, sendo que o declínio dessa esfera pode ser retardado nessa parcela de indivíduos (GOLDSTEIN GC, 2013). Assim sendo, a amostra avaliada no presente estudo pode compor um grupo utilizador de tais tecnologias, em detrimento à prática de atividades físicas, o que pode ter influenciado a correlação entre estas e o nível cognitivo. Outro achado relevante diz respeito à associação entre níveis de atividade física e qualidade de vida. Os resultados obtidos são concordantes com inúmeras pesquisas consistentes que defendem uma relação direta e positiva entre essas duas esferas, como identificado por Phillips SM, et al (2014), em estudo com 321 idosos e por Vagetti GC, et al (2014), em sua revisão de literatura, que ainda subdividiu a variável qualidade de vida em diferentes domínios, observando graus de associação distintos. Pode-se ressaltar, ainda, que o desenho de estudos transversais, como o realizado, sugere apenas a existência de associação causal entre as variáveis trabalhadas, não permitindo estabelecimento de relações temporais comprovadas (FRONTEIRA I, 2013).

Dentre os pontos fortes desse estudo, destaca-se a diversidade de fatores e variáveis analisadas, incluindo-se três instrumentos validados e reconhecidos, além da ficha sociodemográfica, o que permite uma avaliação ampla e abrangente das reais condições inerentes ao contexto do indivíduo idoso. Ademais, a inclusão de quatro pontos de coleta favorece a representatividade da amostra, que retrata de maneira mais fidedigna a população estudada. 
É de suma importância, ainda, enfatizar-se a existência de peculiaridades inerentes às cidades médias, como Divinópolis, em que se recrutou a população do presente estudo. A dinâmica e realidade desse tipo de centro vêm sendo alvo de inúmeras pesquisas de proporções mundiais, sendo que diversas universidades brasileiras desenvolvem trabalhos relacionados a esse tema (ALVES MAS, et al., 2009). O processo de envelhecimento nesse contexto, portanto, é singular, tendo em vista que os serviços públicos, tais como transporte, lazer e o atendimento em saúde são fundamentais para a vida dos idosos e deveriam estar adequados à necessidade dessa população. Entretanto, a implementação das ações e ofertas desses serviços enfrenta o desafio das diferenças e desigualdades entre as regiões do país, que fazem do envelhecimento no Brasil um processo heterogêneo e marcado por perceptíveis diferenças de acordo com o município em que ele ocorre (LIMA LCV, et al., 2016). Considerando-se que há uma tendência nacional de migração dos jovens para a capital e cidades mais populosas, resultando em um envelhecimento dos demais municípios (MELO LA, et al., 2017), torna-se ainda mais evidente a necessidade de uma oferta qualificada desses serviços também nas cidades médias e pequenas.

\section{CONCLUSÃO}

Verificou-se relação positiva entre a qualidade de vida dos idosos e a prática de atividade física, sendo necessário, portanto, destinar maior atenção a essa esfera para a promoção de um envelhecimento mais saudável para a população em geral. Para tal, sugere-se que a gestão pública implante estratégias que suportem e atendam às demandas da população de idosos predominante, cada vez mais envelhecida. Ademais, medidas de investimento em fatores sociodemográficos, como educação, constituem recursos que, a longo prazo, favoreceriam melhores performances cognitivas para as futuras gerações de idosos. Por fim, recomenda-se a realização de novos estudos que obtenham maiores explicações sobre a causalidade das relações observadas, principalmente para a realidade das cidades de médio porte.

\section{REFERÊNCIAS}

1. ALMEIDA AV, et al. A Feminização da Velhice: em foco as características socioeconômicas, pessoais e familiares das idosas e o risco social / The Feminization of Old Age: a focus on the socioeconomic, personal and family characteristics of the elderly and the social risk. Textos \& Contextos, 2015; 14(1): 115, 2015.

2. ALVES MAS, DINIZ AMA. O zoneamento morfológico funcional das cidades médias mineiras: o exemplo de Barão de Cocais. Sociedade \& Natureza, 2009; 20(2):79-91.

3. BENEDETTI TRB, et al. Reprodutibilidade e validade do Questionário Internacional de Atividade Física (IPAQ) em homens idosos. Revista Brasileira de Medicina do Esporte, 2007; 13(1): 11-16.

4. BENNETT IJ, MADDEN DJ. Disconnected Aging: Cerebral White Matter Integrity and Age-Related Differences in Cognition. NIH Public Access, 2009, 6: 247-253.

5. BLESSMANN EJ, GONÇALVES AK. Envelhecimento: equilíbrio, cognição, audição e qualidade de vida. NEIE/UFRGS, 2015 [s.n.].

6. BRASIL. Lei № 8.842, de 4 de janeiro de 1994. Dispõe sobre a política nacional do idoso, cria o Conselho Nacional do Idoso e dá outras providências.

7. BRASIL. Lei No 10.741, de $1^{\circ}$ de outubro de 2003. Dispõe sobre o Estatuto do Idoso e dá outras providências.

8. CARVALHO A, et al. Physical activity and cognitive function in individuals over 60 years of age: A systematic review. Clinical Interventions in Aging, 2014; 9: 661-682.

9. CASTRO C, et al. Influência da escolaridade e condições de saúde no trabalho remunerado de idosos Brasileiros. Ciência e Saude Coletiva [periódico na internet], 2018.

10. DANIEL F, et al. Rastreio cognitivo em estruturas residenciais para pessoas idosas no Concelho de Miranda do Corvo, Portugal. Ciência e Saude Coletiva [periódico da internet], 2018.

11. DIAS EG, et al. Advanced activities of daily living and incidence of cognitive decline in the elderly: the SABE Study. Cadernos de saúde pública, 2015; 31(8):1623-1635.

12. FRONTEIRA I. Observational Studies in the Era of Evidence Based Medicine: Short Review on their Relevance, Taxonomy and Designs. Acta Medica Portuguesa [Internet], 2013; 26(2): 161-170.

13. GOLDSTEIN GC. A. Exergames como recurso de promoção de saúde e envelhecimento ativo. Revista Portal de Divulgação, 2013: p. 21-28. 
14. HARADA CN, LOVE MCN, TRIEBEL K. NIH Public Access. Clinics in Geriatric Medicine.,2013; 29(4): 737-752.

15. INSTITUTO BRASILEIRO DE GEOGRAFIA E ESTATÍSTICA. Censo demográfico, 2010.

16. INSTITUTO BRASILEIRO DE GEOGRAFIA E ESTATÍSTICA. Síntese de indicadores sociais, $2016 ; 39$ [s.n]: 1-63.

17. KISSAKI PT, et al. O impacto da participação em Universidade Aberta à Terceira Idade no desempenho cognitivo. Revista Temática Kairós Gerontologia, 2010; 15(7): 71-87.

18. LANGLOIS F, VU T. Benefits of physical exercise training on cognition and quality of life in frail older adults. The Journals of Gerontology Series B: Psychological Sciences and Social Sciences, 2013; 68 [s.n]: 400-404.

19. LIMA LCV de, VILELLA WV, BITTAR CML. Satisfação de Idosos Residentes em Municípios de Pequeno Porte com os Serviços Públicos Ofertados. Saúde e Pesquisa, 2016; 9(1): 31.

20. LOURENÇO RA, VERAS RP. Mini-Exame do Estado Mental: características psicométricas em idosos ambulatoriais. Revista de Saúde Pública, 2006; 40(4): 712-719.

21. LUZ EP da, et al. Perfil sociodemográfico e de hábitos de vida da população idosa de um município da região norte do Rio Grande do Sul, Brasil. Revista Brasileira de Geriatria e Gerontologia., 2014; 17(2): 303-314.

22. MACHADO JC, et al. Declínio cognitivo de idosos e sua associação com fatores epidemiológicos em Viçosa, Minas Gerais. Revista Brasileira de Geriatria e Gerontologia, 2011: 109-121.

23. MELO DM de, BARBOSA AJG. O uso do Mini-Exame do Estado Mental em pesquisas com idosos no Brasil: uma revisão sistemática. Ciência \& Saúde Coletiva, 2015; 20(12): 3865-3876.

24. MELO LA de, et al. Socioeconomic, regional and demographic factors related to population ageing. Revista Brasileira de Geriatria e Gerontologia, 2017; 20(4): 493-501.

25. NAQVI R, et al. Preventing cognitive decline in healthy older adults. Canadian Medical Association Journal, 2013; 185(10): 881-885.

26. OLIVEIRA D, et al. O nível de atividade física como um fator interveniente no estado cognitivo de idosos da atenção básica a saúde.. Ciência e Saude Coletiva [periódico na internet], 2018.

27. PHILLIPS SM, WÓJCICKI TR, MCAULEY E. Physical Activity and Quality of Life in Older Adults: An 18- Month Panel Analysis. Quality of Life Research, 2014; 22(7): 1647-1654.

28. SANTOS S, et al. Avaliação da confiabilidade do mini-exame do estado mental em idosos e associação com variáveis sociodemográficas. Cogitare Enfermagem, 2010; 15(3): 406-412.

29. SATOH M, et al. The Effects of Physical Exercise with Music on Cognitive Function of Elderly People: Mihama-Kiho Project. PLoS ONE, 2014; 9(4): e95230.

30. ORGANIZAÇÃO MUNDIAL DA SAÚDE. Manual WHOQOL-OLD. UFRGS [Internet], 2005: 14-19.

31. SILVEIRA MM da. Desempenho Cognitivo, estado emocional, qualidade de vida e habilidade motora manual de idosos participantes de oficinas de informática. PUCRS, 2015.

32. SOARES RM, DINIZ AB, CATTUZZO MT. Associação entre atividade física, aptidão física e desempenho cognitivo em idosos. Motricidade, 2013; 9(2): 84-93.

33. VAGETTI GC, et al. Association between physical activity and quality of life in the elderly: a systematic review, 20002012. Revista Brasileira de Psiquiatria, 2014; 36(1): 76-88. 